\title{
The ubiquitin-like modifier FAT10 in antigen processing and antimicrobial defense
}

\author{
Michael Basler ${ }^{\mathrm{a}, \mathrm{b}, *}$, Stefanie Buerger ${ }^{\mathrm{a}}$, Marcus Groettrup ${ }^{\mathrm{a}, \mathrm{b}, * *}$ \\ a Division of Immunology, Department of Biology, University of Konstanz, D-78457 Konstanz, Germany \\ ${ }^{\mathrm{b}}$ Biotechnology Institute Thurgau (BITg) at the University of Konstanz, CH-8280 Kreuzlingen, Switzerland
}

Keywords:

Antigen presentation

Antigen processing

Ubiquitin-like modifier

FAT10

\section{A B S T R A C T}

The ubiquitin-like modifier (ULM) HLA-F adjacent transcript 10 (FAT10) is encoded in the MHC locus, is up-regulated during dendritic cell maturation, is highly expressed in lymphoid tissues, and strongly induced by interferon (IFN)- $\gamma$ and tumor necrosis factor (TNF)- $\alpha$. FAT10 is the only ULM known to date which directly targets its hundreds of substrates for degradation by the proteasome. This implies a role for FAT10 in antigen presentation. Indeed, fusion of FAT10 to viral proteins enhanced their presentation along the proteasome dependent MHC class I presentation pathway. In this review we discuss the FAT10 conjugation system as an alternative and distinct pathway for MHC class I and II antigen processing. Furthermore, we review the recent finding that FAT10 plays a role in antimicrobial defense against intracellular pathogens.

\section{Introduction}

Ubiquitin-like modifiers (ULM) post-translationally modify cellular targets in pathways that are parallel to but distinct from the ubiquitin system and influence diverse biological processes. The covalent modification with the cytokine-inducible ULM HLA-F adjacent transcript 10 (FAT10) targets proteins in a ubiquitinindependent manner for proteasomal degradation (Raasi et al., 2001; Hipp et al., 2005; Schmidtke et al., 2009). As other ULMs, FAT10 is conjugated to its substrates via isopeptide linkage mediated by an E1, E2, and possibly E3 enzyme cascade, where UBA6 (also termed UBE1L2, E1-L2, or MOP-4) and USE1 (UBA6-specific E2 enzyme) serve as E1-type activating and E2-type conjugating enzymes, respectively (Aichem et al., 2010; Pelzer and Groettrup, 2010; Chiu et al., 2007). FAT10 has been implicated in multiple cellular processes like apoptosis, spindle check point control during mitotic cell cycle, and NF- $\kappa$ B activation. Basal FAT10 expression is most prominent in organs of the immune system, like thymus, fetal

\footnotetext{
* Corresponding author at: Department of Biology, Division of Immunology, University of Konstanz, P1101 Universitätsstrasse 10, D-78457 Konstanz, Germany. Tel.: +49 7531882258 ; fax: +49 7531883102 .

** Corresponding author at: Department of Biology, Division of Immunology, University of Konstanz, P1101 Universitätsstrasse 10, D-78457 Konstanz, Germany. Tel.: +49 7531 882130; fax: +49 7531883102 .

E-mail addresses: Michael.Basler@uni-konstanz.de (M. Basler), Marcus.Groettrup@uni-konstanz.de (M. Groettrup).
}

liver, lymph nodes, and spleen. In addition, expression of FAT10 can be synergistically induced by the pro-inflammatory cytokines IFN $\gamma$ and TNF $\alpha$ and it is up-regulated during dendritic cell maturation (summarized in (Schmidtke et al., 2014)). Mice lacking FAT10 are viable and fertile, indicating that housekeeping functions are not grossly influenced by FAT10 (Canaan et al., 2006). Nevertheless, lymphocytes of FAT10 knockout mice are more prone to spontaneous apoptotic death and mice demonstrated a high level of sensitivity towards endotoxin challenge (Canaan et al., 2006). Furthermore, FAT10-deficient mice have an extended lifespan and reduced adiposity (Canaan et al., 2014).

\section{FAT10 in antigen processing}

The major histocompatibility complex (MHC) class-I restricted pathway of antigen processing allows the presentation of intracellular antigens to cytotoxic $\mathrm{T}$ lymphocytes. The main protease involved in this process is the proteasome (Basler et al., 2009). Proteins destined for degradation by the 26S proteasome are usually marked with ubiquitin, which requires the coordinated activities of ubiquitin-activating (E1), -conjugating (E2), and -ligating (E3) enzymes. It is generally assumed that ubiquitin is the main driver for proteasomal degradation in MHC-I antigen processing, although experimental evidence for a role of ubiquitin in this process is rather limited and contradictory (Michalek et al., 1993; Cox et al., 1995). In a recent study, the knockdown of ubiquitin, expression of a lysine 48 (K48) ubiquitin mutant, or inhibition of proteasome 


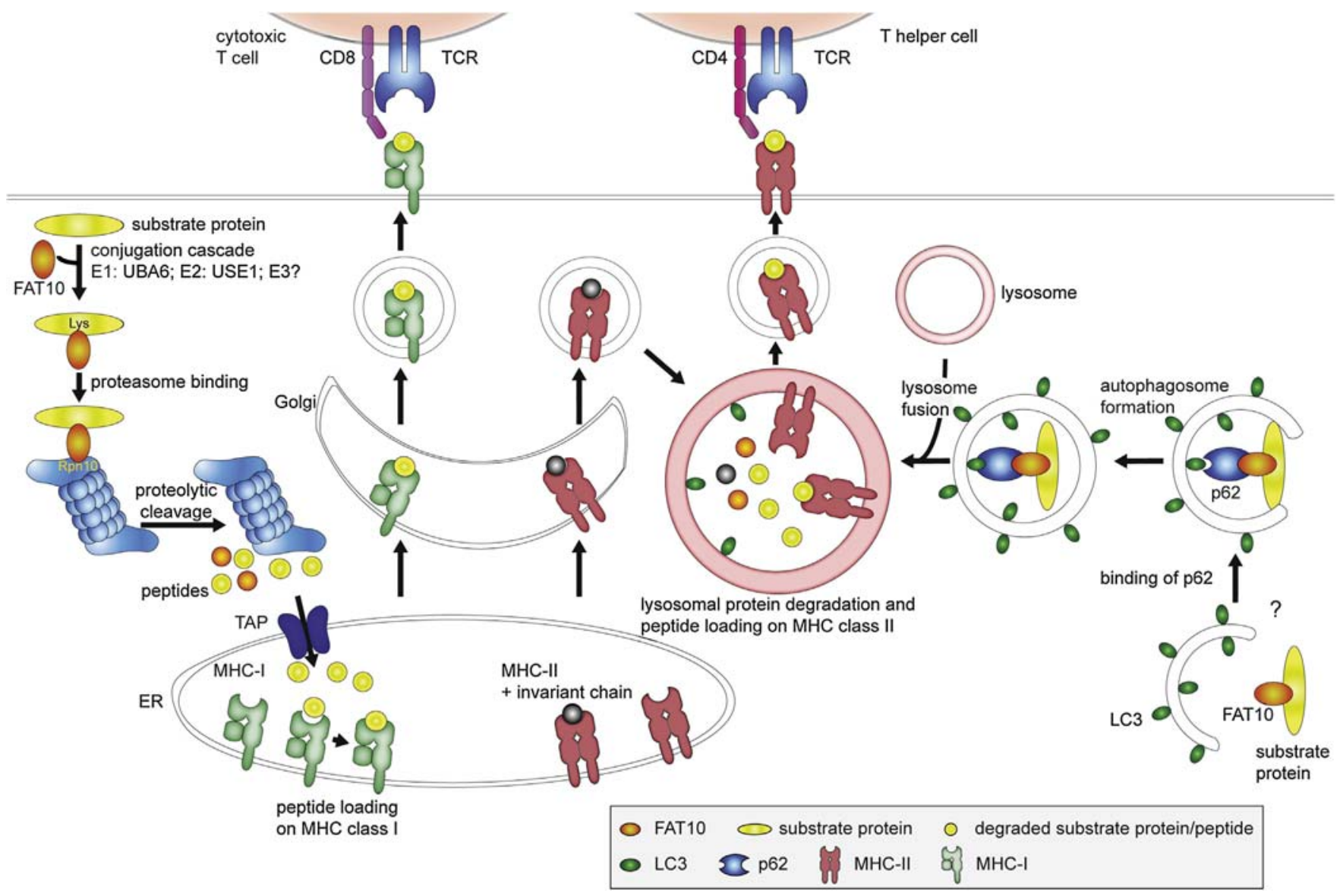

Fig. 1. FAT10 in MHC class I and MHC class II antigen presentation. FAT10 conjugation occurs in a catalytical cascade via an E1 enzyme (UBA6), E2 enzyme (USE1) and so far unidentified E3 enzymes. This results in the formation of an isopeptide bond between FAT10 and a lysine of the substrate protein. Docking of FAT10 and its substrate protein to the proteasome occurs via the VWA domain of the 19S proteasome regulator subunit Rpn10. FAT10 and its substrate protein are degraded by the proteasome. The peptides are subsequently transferred into the ER-lumen via the transporter associated with antigen processing (TAP) where they are loaded on empty MHC class I molecules. The assembled peptide-MHC complexes are then transported to the cell surface via the ER-Golgi network where they are presented to CD ${ }^{+} \mathrm{T}$ cells. The so far hypothetical involvement of FAT10 in the MHC class II antigen presentation pathway involves binding of FAT10ylated substrate proteins to the autophagy adapter p62. Interaction of p62 with LC3 leads to autophagosome formation and subsequent fusion with lysosomes. MHC class II molecules containing an invariant chain blocking the peptide binding groove are exported via the ER-Golgi network. Upon fusion of the vesicles with the autophagolysosomes, lysosomal proteases degrade the invariant chain as well as the FAT10 substrate. Peptides derived from FAT10 substrate proteins can then bind to MHC-II and peptide-MHC complex are exported to the cell surface for presentation to CD4 $4^{+}$T cells.

associated deubiquitinases significantly impaired antigen presentation (Fiebiger et al., 2015). The authors suggested a model in which the binding of the antigen substrate by an adaptor protein leads to K48-poly-ubiquitylation of the adaptor (but not the antigen) and the subsequent delivery of the antigen cargo for degradation by the $26 \mathrm{~S}$ proteasome. In another study, using an inducible vaccinia virus system overexpressing wild type and dominantnegative lysineless forms of ubiquitin in mammalian cells the role of ubiquitin in antigen processing was assessed (Huang et al., 2011). Thereby, ubiquitin-dependent degradation appeared to play a major role in the processing of ER-targeted proteins, but there was a rather restricted role in the processing of cytosolic proteins. The presence of such functionally distinct pathways suggests the existence of alternative proteasomal targeting systems, like ULM, involved in antigen presentation. Since FAT10 is the only ULM able to directly target proteins for proteasomal degradation, it is likely that FAT10 might be involved in an alternative proteasomal targeting for MHC-I restricted presentation. Furthermore, like several components involved in antigen presentation FAT10 is encoded in the MHC locus. Therefore, it seems likely that FAT10-mediated proteasomal degradation might influence MHC-I antigen presentation (Fig. 1).
FAT10 was described as a ubiquitin-independent signal for proteasomal degradation that directly targets proteins for degradation by the proteasome (Hipp et al., 2005; Schmidtke et al., 2009). The degradation of FAT10-linked proteins by the proteasome is strongly accelerated by the ubiquitin-like-ubiquitin-associated protein NEDD8 ultimate buster-1 long (NUB1L) (Hipp et al., 2004). To initiate proteolysis both FAT10 and NUB1L dock to the $26 \mathrm{~S}$ proteasome. Docking of FAT10 to the 26S proteasome occurs via the VWA domain of the 19S cap of the 26S proteasome subunit Rpn10 (S5a), whereas NUB1L can bind to both 19S cap subunits Rpn10 and Rpn1/S2 (Rani et al., 2012). Hence, in principle, FAT10 is able to target potential antigens for proteasomal degradation. Indeed, a role of FAT10 in MHC antigen presentation has been demonstrated (Ebstein et al., 2012; Schliehe et al., 2012). Ebstein et al. (2012) expressed fusion proteins consisting of the human cytomegalovirus (HCMV)-derived pp65 antigen N-terminally tagged with either ubiquitin or FAT10 in HeLa cells and analyzed the presentation of the immunodominant pp65495-503 epitope in these cells. In comparison to the untagged pp65, the FAT10-pp65 fusion protein enhanced antigen presentation approximately twofold, a similar extent as that seen with the ubiquitin-pp65 fusion protein. The improved pp65495-503 presentation obtained with ubiquitin-pp65 
was substantially reduced when all the seven lysine residues of the fused ubiquitin moiety were changed into arginine residues indicating that Ub-pp65 relies on the poly-ubiquitylation of its $\mathrm{N}$ terminal ubiquitin. Nevertheless, it was not addressed whether the enhanced FAT10-induced antigen presentation is dependent on poly-FAT10ylation. However, in a different study, not addressing antigen presentation, FAT10 with its two ubiquitin-like domains served as a degradation signal with no need for chain formation (Rani et al., 2012). Rpn10 knockdown was associated with a significantly impaired pp65495-503 epitope presentation from the FAT10-pp65 fusion protein, indicating that docking of FAT10 to the $26 S$ proteasome occurs via Rpn10 (Ebstein et al., 2012). Interestingly, FAT10-pp65 processing is not altered by immunoproteasome subunits and PA28 $\alpha / \beta$, the IFN $\gamma$ inducible components of the proteasome. An involvement of FAT10 in MHC antigen presentation was also demonstrated by Schliehe et al. (2012). N-terminal fusion of the lymphocytic choriomeningitis virus (LCMV) nucleoprotein (NP) with ubiquitin (Ub-NP) or FAT10 (FAT10-NP) enhanced its degradation rate. The N-terminal fusion of the NP to either ubiquitin or FAT10 converted the long-lived NP into a rapidly degraded FAT10-NP fusion protein. Transfection of cells with these constructs increased the degradation rate of FAT10-NP leading to a 2-3 fold enhanced presentation of the nucleoprotein-derived MHC class I epitopes $\mathrm{NP}_{396-404}$ or $\mathrm{NP}_{118-126}$. Additionally, infection of cells with a recombinant vaccinia virus expressing FAT10-NP in vitro strongly increased the presentation of the LCMV-derived $\mathrm{NP}_{396-404}$ or $\mathrm{NP}_{118-126}$. Interestingly, the enhanced presentation did not improve the cytotoxic T cell (CTL) response to these epitopes in mice immunized with FAT10-NP, neither in a DNA immunization set-up nor after infection with recombinant vaccinia viruses. Instead, the NP specific CTL response correlated positively with the stability of the antigen, with stable antigens showing the strongest CTL response. As stable antigen in antigen donor cells is required for efficient cross-presentation, this result was interpreted as evidence for cross-presentation for priming NP-specific CTL responses. Taken together, these results strongly suggest that linkage to FAT10 can feed antigens into the MHC class I antigen processing pathway. DC aggresome-like structures (DALIS) contain poly-ubiquitylated proteins. These transient aggresome-like structures function as storage compartments of antigenic proteins during the maturation of dendritic cells and peptides derived from DALIS can be presented by MHC class I molecules (Pierre, 2005). Kalveram et al. (2008) reported that FAT10 interacts with the cytoplasmic protein histone deacetylase 6 (HDAC6) and localizes to aggresomes under proteasome inhibition. Hence, it remains to be determined whether FAT10 is also involved in the formation of DALIS and thereby influences class I presentation.

Apart from a role in MHC class I antigen presentation, FAT10 might influence class II presentation (Fig. 1). Classically, endocytosis delivers non self-antigens to MHC-II molecules, but a substantial portion of MHC class II ligands originates from cytosolic and nuclear antigens. Autophagy is an evolutionarily conserved pathway with nutrient-recycling functions during starvation, however, in higher eukaryotes this pathway is also used to present peptides on MHC class II molecules to CD4 ${ }^{+} \mathrm{T}$ cells (Munz, 2012). It has been demonstrated that the autophagosomal receptor p62/SQSTM1 becomes covalently mono-FAT10ylated at several lysines, and that FAT10 co-localizes with p62 in p62 bodies (Aichem et al., 2012). Furthermore, FAT10 is involved in the formation of Mallory-Denk bodies, which are aggresome-like structures in hepatocellular carcinoma cells composed of ubiquitylated proteins (French et al., 2012). Additionally, FAT10 decorates autophagy-targeted Salmonella and contributes to Salmonella resistance in mice (Spinnenhirn et al., 2014)(see below). Hence, the association of FAT10 with autophagosomal markers indicates that FAT10 might, similar to class I presentation, be involved in MHC class II presentation. Supporting this idea is the fact that FAT10 is highly expressed in the thymus (Lukasiak et al., 2008), an organ in which autophagy is an essential process in mediating tolerance of $\mathrm{CD} 4^{+} \mathrm{T}$ cells. In humans and in rats type 1 diabetes (T1D) is strongly associated with a permissive class II MHC haplotype, indicating that class II antigen presentation plays a crucial role in this autoimmune disease. Recently, the fat10 gene (also called ubiquitin D (ubd) gene) could be linked to enhanced susceptibility to virus-triggered autoimmune diabetes in rats (Cort et al., 2014). Gene expression profiling of pancreatic lymph nodes in susceptible and resistant rats during disease induction showed differences in fat10 transcript abundance. Furthermore, LEW.1WR1 rats lacking FAT10 expression showed a reduced susceptibility to virus-induced T1D. These results strongly support the notion of a role for FAT10 in class II antigen presentation.

\section{FAT10 in antimicrobial defense}

Invasion of bacteria into eukaryotic cells is counteracted by cell autonomous innate immune mechanisms including xenophagy. The initiation of the destruction of intracellular bacteria by xenophagy is mediated by the decoration of cytosolic bacteria with ubiquitin and binding of galectin-8. Whether pathogens are either directly ubiquitylated or whether ubiquitylated host proteins accumulate on their surface to initiate xenophagy is still a matter of debate. Ubiquitin decoration of cytosolic bacteria leads to the recruitment of autophagy adaptors like p62, NDP52, and optineurin. FAT10, via an UBA-domain independent covalent interaction, can be conjugated to the autophagy adaptor p62 (Aichem et al., 2012). Additionally, a strong non-covalent interaction between FAT10 and p62 has been described (Aichem et al., 2012). Functioning as an autophagosomal adaptor between ubiquitin on bacteria and the nascent autophagosome-linked protein LC3, p62 has been implicated in the capture of cytosolic bacteria. This indicates that FAT10 might, analogous to ubiquitin, be involved in targeting pathogens for autophagosomal degradation. Indeed, FAT10 decorated cytosolic Salmonella typhimurium which were targeted for autophagy (Spinnenhirn et al., 2014). Similar to ubiquitin, FAT10 decoration and autophagosomal targeting of S. typhimurium occurred with the same kinetics. Nevertheless, the percentage of ubiquitin and FAT10 decorated bacteria markedly differed, suggesting that the FAT10-targeted structures are not the same as the ubiquitylated ones. Unexpectedly, neither FAT10 deficiency nor overexpression did significantly change bacterial replication in vitro. Nevertheless, infection of a mouse strain susceptible to S. typhimurium revealed a role of FAT10 in antimicrobial defense. 14 days post infection, a significantly enhanced bacterial load in mesenteric lymph nodes from FAT10-deficient mice as compared to wild type mice was observed (Spinnenhirn et al., 2014). Additionally, FAT10-deficient mice showed a tendency to lose more body weight than their FAT10-proficient wild type controls. Furthermore, survival experiments following $S$. typhimurium infection of susceptible wild type and FAT10-deficient mice resulted in a higher rate of death events in mice lacking FAT10. The late effect of FAT10 deficiency on Salmonella infection in mice could also indicate a role in adaptive immunity, but this remains to be determined. Taken together, FAT10 decorates S. typhimurium in cells and contributes to the protection of mice against this pathogen.

\section{Conclusions}

The FAT10 axis was described as a novel route for MHC class I antigen presentation (Ebstein et al., 2012; Schliehe et al., 2012). FAT10 is the only ubiquitin-like modifier known to date, which, like poly-ubiquitin chains, can directly target several substrates for degradation by the 26S proteasome (Schmidtke et al., 2014). 
Although the ubiquitin-like modifier FAT10 is in many aspects similar to ubiquitin, the expression of FAT10 upon cytokine stimulation suggests that this novel route may allow presentation of different antigenic peptides or increased amounts of peptides in inflamed tissue compared to the classical ubiquitin conjugation system. E3 ligases are crucial in determining substrate specificity in the ubiquitin-proteasome system. Hence, E3 ligases exclusively involved in FAT10 conjugation could target a different pool of proteins for degradation and thereby alter the peptides presented on MHC-I. However, so far, no E3 ligases in the FAT10 conjugation machinery have been identified. To investigate whether FAT10 E3 ligases, compared to ubiquitin, target different proteins, the discovery of E3 ligases in the FAT10 conjugation process is highly warranted. Docking of FAT10 to the $26 \mathrm{~S}$ proteasome occurs via the VWA domain of Rpn10 (S5a) while poly-ubiquitin binds to the ubiquitin-interaction motifs of Rpn10 as well as the subunit Rpn13 (Rani et al., 2012; Ebstein et al., 2012). This might result in a differential processing of FAT10 as compared to ubiquitin-conjugated proteins by the $26 \mathrm{~S}$ proteasome. Furthermore, FAT10 might target short-lived proteins for degradation, and thereby be involved in the presentation of defective ribosomal products (DRIPs). Taken together, it seems that the FAT10-conjugation machinery might represent a complementary pathway when ubiquitin becomes a rate-limiting factor. If the proteasome is overwhelmed or inhibited, both ubiquitin and FAT10 accumulate in aggresomes (Kalveram et al., 2008). Additionally, FAT10 interacts with the autophagosomal receptor p62/SQSTM1 (Aichem et al., 2012). This suggests that FAT10 might be involved in MHC class II presentation. However, further experiments are needed to elucidate the exact role of FAT10 in class II presentation. FAT10-deficient mice have apart from minor alterations no obvious phenotype (Canaan et al., 2006, 2014). Nevertheless, FAT10-deficient mice revealed a higher susceptibility for S. typhimurium suggesting a role for FAT10 in the intracellular defense against bacteria (Spinnenhirn et al., 2014). Hence, further investigations with different pathogens are warranted to clarify the biological role of the ubiquitin-like modifier FAT10.

\section{Conflict of interest}

The authors have no financial conflicts of interest.

\section{Acknowledgments}

This work was funded by the German Research Foundation grant Nr. BA 4199/2-1 to M.B. and GR 1517/2.4 and GR $1517 / 10-2$ to M.G.

\section{References}

Aichem, A., Pelzer, C., Lukasiak, S., Kalveram, B., Sheppard, P.W., Rani, N., Schmidtke, G., Groettrup, M., 2010. USE1 is a bispecific conjugating enzyme for ubiquitin and FAT10, which FAT10ylates itself in cis. Nat. Commun. 1,13.
Aichem, A., Kalveram, B., Spinnenhirn, V., Kluge, K., Catone, N., Johansen, T., Groettrup, M., 2012. The proteomic analysis of endogenous FAT10 substrates identifies p62/SQSTM1 as a substrate of FAT10ylation. J. Cell Sci. 125, 4576-4585.

Basler, M., Lauer, C., Beck, U., Groettrup, M., 2009. The proteasome inhibitor bortezomib enhances the susceptibility to viral infection. J. Immunol. 183,6145-6150.

Canaan, A., Yu, X., Booth, C.J., Lian, J., Lazar, I., Gamfi, S.L., Castille, K., Kohya, N., Nakayama, Y., Liu, Y.C., et al., 2006. FAT10/diubiquitin-like protein-deficient mice exhibit minimal phenotypic differences. Mol. Cell. Biol. 26, 5180-5189.

Canaan, A., DeFuria, J., Perelman, E., Schultz, V., Seay, M., Tuck, D., Flavell, R.A., Snyder, M.P., Obin, M.S., Weissman, S.M., 2014. Extended lifespan and reduced adiposity in mice lacking the FAT10 gene. Proc. Natl. Acad. Sci. U. S. A. 111, 5313-5318.

Chiu, Y.H., Sun, Q., Chen, Z.J., 2007. E1-L2 activates both ubiquitin and FAT10. Mol. Cell 27, 1014-1023.

Cort, L., Habib, M., Eberwine, R.A., Hessner, M.J., Mordes, J.P., Blankenhorn, E.P., 2014. Diubiquitin (Ubd) is a susceptibility gene for virus-triggered autoimmune diabetes in rats. Genes Immun. 15, 168-175.

Cox, J.H., Galardy, P., Bennink, J.R., Yewdell, J.W., 1995. Presentation of endogenous and exogenous antigens is not affected by inactivation of E1 ubiquitin-activating enzyme in temperature-sensitive cell lines. J. Immunol. 154, 511-519.

Ebstein, F., Lehmann, A., Kloetzel, P.M., 2012. The FAT10- and ubiquitin-dependent degradation machineries exhibit common and distinct requirements for $\mathrm{MHC}$ class I antigen presentation. Cell. Mol. Life Sci. 69, 2443-2454.

Fiebiger, B.M., Pfister, H., Behrends, U., Mautner, J., 2015. Polyubiquitination of lysine-48 is an essential but indirect signal for MHC class I antigen processing. Eur. J. Immunol. 45, 716-727.

French, S.W., French, B.A., Oliva, J., Li, J., Bardag-Gorce, F., Tillman, B., Canaan, A., 2012. FAT10 knock out mice livers fail to develop Mallory-Denk bodies in the DDC mouse model. Exp. Mol. Pathol. 93, 309-314.

Hipp, M.S., Raasi, S., Groettrup, M., Schmidtke, G., 2004. NEDD8 ultimate buster-1L interacts with the ubiquitin-like protein FAT10 and accelerates its degradation. J. Biol. Chem. 279, 16503-16510.

Hipp, M.S., Kalveram, B., Raasi, S., Groettrup, M., Schmidtke, G., 2005. FAT10, ubiquitin-independent signal for proteasomal degradation. Mol. Cell. Biol. 25, 3483-3491.

Huang, L., Marvin, J.M., Tatsis, N., Eisenlohr, L.C., 2011. Cutting edge: selective role of ubiquitin in MHC class I antigen presentation. J. Immunol. 186, 1904-1908.

Kalveram, B., Schmidtke, G., Groettrup, M., 2008. The ubiquitin-like modifier FAT10 interacts with HDAC6 and localizes to aggresomes under proteasome inhibition. J. Cell Sci. 121, 4079-4088.

Lukasiak, S., Schiller, C., Oehlschlaeger, P., Schmidtke, G., Krause, P., Legler, D.F., Autschbach, F., Schirmacher, P., Breuhahn, K., Groettrup, M., 2008. Proinflammatory cytokines cause FAT10 upregulation in cancers of liver and colon. Oncogene 27, 6068-6074.

Michalek, M.T., Grant, E.P., Gramm, C., Goldberg, A.L., Rock, K.L., 1993. A role for the ubiquitin-dependent proteolytic pathway in MHC class I-restricted antigen presentation. Nature 363, 552-554.

Munz, C., 2012. Antigen processing for MHC class II presentation via autophagy. Front. Immunol. 3, 9

Pelzer, C., Groettrup, M., 2010. FAT10: activated by UBA6 and functioning in protein degradation. Subcell. Biochem. 54, 238-246.

Pierre, P., 2005. Dendritic cells, DRiPs, and DALIS in the control of antigen processing. Immunol. Rev. 207, 184-190.

Raasi, S., Schmidtke, G., Groettrup, M., 2001. The ubiquitin-like protein FAT10 forms covalent conjugates and induces apoptosis. J. Biol. Chem. 276, 35334-35343.

Rani, N., Aichem, A., Schmidtke, G., Kreft, S.G., Groettrup, M., 2012. FAT10 and NUB1L bind to the VWA domain of Rpn10 and Rpn1 to enable proteasome-mediated proteolysis. Nat. Commun. 3, 749.

Schliehe, C., Bitzer, A., van den Broek, M., Groettrup, M., 2012. Stable antigen is most effective for eliciting CD8+ T-cell responses after DNA vaccination and infection with recombinant vaccinia virus in vivo. J. Virol. 86, 9782-9793.

Schmidtke, G., Kalveram, B., Groettrup, M., 2009. Degradation of FAT10 by the 265 proteasome is independent of ubiquitylation but relies on NUB1L. FEBS Lett. 583 , 591-594.

Schmidtke, G., Aichem, A., Groettrup, M., 2014. FAT10ylation as a signal for proteasomal degradation. Biochim. Biophys. Acta 1843, 97-102.

Spinnenhirn, V., Farhan, H., Basler, M., Aichem, A., Canaan, A., Groettrup, M., 2014 The ubiquitin-like modifier FAT10 decorates autophagy-targeted Salmonella and contributes to Salmonella resistance in mice. J. Cell Sci. 127, 4883-4893. 